\title{
RESVERATROL AS A POTENTIAL AGENT FOR NEUROPROTECTION AGAINST INTRACEREBRAL HEMORRHAGE: INSIGHT ON THE ROLE OF ADENOSINE A1 RECEPTORS
}

\author{
I. lezhitsa' ${ }^{1,2}$, N.A.W. Abd. Aziz³ ${ }^{3}$ R. Agarwal' ${ }^{1}$ A. Abd. Latiff ${ }^{3}$ and N.M. Ismail' ${ }^{1}$ \\ ${ }^{1}$ International Medical University, IMU Clinical School, Seremban, Malaysia. \\ ${ }^{2}$ Volgograd State Medical University, Research Centre for Innovative Medicines, Russia. \\ ${ }^{3}$ Centre for Neuroscience Research, Faculty of Medicine, Universiti Teknologi MARA, Sungai \\ Buloh Campus, Selangor, Malaysia.
}

DOI: 10.19163/MedChemRussia2021-2021-88

E-mail: Igorlezhitsa@imu.edu.my

Stroke is a common cause of brain injury and contributes to significant mortality worldwide. Intracerebral hemorrhage $(\mathrm{ICH})$ remains the least treatable stroke and is characterized by hematoma (HT) expansion and perihematomal (PHT) damage. Tumor necrosis factor- $\alpha$ (TNF- $\alpha$ ) activation underlies PHT damage in ICH. TNF- $\alpha$ triggers apoptosis and necroptosis by increasing caspase- 3 (CASP3) and receptor-interacting protein kinase 3 (RIPK3) respectively. None of the current therapy protects against PHT. The only definite treatment is by evacuation of HT, which often results in incomplete recovery from HT-induced secondary injury. In view of the neuroprotective effects of trans-resveratrol (RV), we investigated the effects of RV on brain injury induced by collagenase (COL) in Sprague-Dawley rats. The objective of this study was to elucidate the neuroprotective effect and mechanisms of action of $\mathrm{RV}$, with an insight on the role of adenosine $\mathrm{A} 1$ receptor (A1R), against COLinduced ICH in rats. In this study, rats were separated into 5 groups: (i) control, (ii) sham-operated rats, (iii) ICH rats pretreated with vehicle (0.1\% DMSO saline, i.c.v.), (iv) ICH rats pretreated with RV (4 nmol, i.c.v.), and (v) ICH rats pretreated with RV (4 nmol) and the A1R antagonist, DPCPX $(8 \mathrm{nmol}$, i.c.v.). Thirty minutes after pretreatment, ICH was induced by intrastriatal injection of COL (0.04 U). Forty-eight hours after $\mathrm{ICH}$, rats were subjected to a variety of neurological and neurobehavioural tests and the brain tissues were processed for gross, histological and biochemical analyses. Sections of brain tissue were examined for HT volume through gross morphometry, while morphological features, midline shift, damaged area and neuronal density through histopathological examination using $\mathrm{H} \& \mathrm{E}$ and neuronal nuclei (NeuN) immunostaining. This study revealed that pretreatment with $\mathrm{RV}$ reduced HT volume, damaged area and neuronal depletion. DPCPX abolished effects by RV on damaged area and neuronal depletion. Overall, our data demonstrated that RV resolved HT and preserved PHT area, while A1R is involved in the neuroprotective effects by RV in PHT damage. In the next part of our study, mechanisms of neuroprotection by RV against COL-induced PHT damage in rats was elucidated. Homogenate of PHT brain tissues were examined for expression of TNF- $\alpha$, CASP3 and RIPK3 activity using ELISA, while A1R, mitogen-activated protein kinase P38 (MAPK P38), heat shock protein (Hsp90), brain-derived neurotrophic factor (BDNF) and tropomyosin-related kinase B (TrkB) expression using RT-qPCR. It was observed that pretreatment with RV restored the expression of TNF- $\alpha$, CASP3, RIPK3, A1R and Hsp90, and increased transcriptional activity of BDNF/ TrkB. DPCPX abolished effects by RV on CASP3, RIPK3, A1R, Hsp90 and BDNF. Overall, our data demonstrated that $A 1 R$ is involved in the antiapoptotic and antinecroptotic effects of RV in PHT area via upregulation of BDNF. The final study was done to evaluate the sensorimotor function of rats using neurological severity score, tail flick, open field, grip strength and rotarod tests. This part of study demonstrated that RV protected against signs of neurological deficit including general locomotor, forelimb activity and motor coordination, and partially preserved behavioural activities. DPCPX abolished the effects of RV. This data demonstrated that A1R is involved in the effects by RV against COL-induced neurological and neurobehavioural deficits in rats. In conclusion, this study demonstrates involvement of A1R in the mechanisms of neuroprotection by RV by inhibiting CASP3dependent apoptosis and RIPK3-dependent necroptosis in PHT area, probably via the cross talk between $A 1 R$ and BDNF/TrkB pathway. 